\title{
EDITOR'S PAGE
}

\section{Cervical SCI patients treated by modest hypothermia}

\author{
JJ Wyndaele \\ Antwerp University Hospital, Antwerp, Belgium \\ E-mail: wyndaelejj@skynet.be
}

Dear Spinal Cord reader,

The very sad news has reached us that Mr. P Harris, the second editor in chief of Spinal Cord Journal has passed away. I have known him well and appreciated him a lot. Our sincere condolences to his wife Sheelah and his family. An obituary is being prepared to be published soon.

The review manuscript of this issue evaluated the Walking Index for Spinal Cord Injury (WISCI II). John Ditunno et al. underline its unique characteristics as a capacity measure of walking function and its strong metric properties. Appropriate use of the WISCI II was clarified and incorporated into a new guide for its use.

Arazpour et al. compared the energy expenditure during walking with mechanical orthoses (hip knee ankle foot orthosis/HKAFO, and the isocentric reciprocating gait orthosis/IRGO) with a powered gait orthosis (PGO) in individuals with SCI.

Chan et al. found cervical spine (CS) injuries to be prevalent in a retrospective review of 365 paediatric severe trauma patients (0-17 years), defined as an Injury Severity Score 12, admitted to the Paediatric Intensive Care Unit. Attention to CS collar management protocols is needed as $10 \%$ complications were found.

Phontee et al. found that $1 / 3$ of independent ambulatory subjects with SCI experienced falls, especially those with better functional ability. Thus, apart from emphasizing the ability to walk independently, rehabilitation professionals may need to seek strategies that improve balance and safety.

Gross et al. showed, in a retrospective study, that in traumatic SCI individuals, a tethered cord can be responsible for clinical and radiological changes which are strictly localized to the perilesional area. The term perilesional myeloradiculopathy is proposed for this complication, which requires cord release surgery.

Vasquez et al. found that discrepancies between light touch (LT) and pin prick (PP) could relate to the greater complexity of the PP test or a difference in the extent of injury to the posterior columns (LT) and spinothalamic (PP) tracts.

Pramodhyakul et al. indicate that obstacle crossing training immediately enhanced functional ability related to walking of ambulatory participants with SCI. A further longitudinal, randomized controlled trial is needed to support this.

Two Korean contributions on transanal irrigation by Choi et al. in spina bifida children and by Kim et al. in SCI individuals.

Dididze et al. present the largest study cohort of cervical SCI patients treated by modest hypothermia to date.

Chen et al. evaluated autonomic dysreflexia (AD) during urodynamics. Aging may decrease AD symptoms and the magnitude of DBP elevation, possibly through the mechanism of decreased baroreceptor sensitivity. Monitoring is strongly suggested during the tests.

More interesting studies in this SC May issue: Erhan et al. on elbow joint; Equebal et al. on the relationship between gender, age, injury-related variables and rehabilitation outcomes in India; Cao et al. in a huge cohort on the relationship of biographic and injury characteristics with mortality; Stöhrer comparing propiverine ER $45 \mathrm{mg}$ s.i.d. and propiverine IR $15 \mathrm{mg}$ t.i.d for neurogenic bladder treatment; Wong et al. on a validation of the Screening Tool for the Assessment of Malnutrition in Paediatric (STAMP).

Enjoy reading and do not hesitate to contact us with questions, suggestions or else. 